\title{
Endoscopic Intervention through Endoscopic Retrograde Cholangiopancreatography in the Management of Symptomatic Pancreas Divisum: A Long-Term Follow-Up Study
}

\author{
Yi Lu, Bin Xu, Lu Chen, Li-ke Bie, and Biao Gong \\ Digestive Endoscopy Center, Department of Gastroenterology, Ruijin Hospital, Shanghai Jiaotong University School of Medicine, Shanghai, China
}

Background/Aims: To evaluate the efficacy and safety of endoscopic retrograde cholangiopancreatography (ERCP) for the treatment of symptomatic pancreas divisum (PD) and to discuss whether ERCP procedures and outcomes in younger patients differ from those of adults. Methods: Symptomatic patients with PD were included in the study and divided into underaged (age $\leq 17$ years) and adult (age $\geq 18$ years) group. The clinical information of each patient was reviewed, and then the patients were contacted by telephone or their medical records were reviewed to determine their long-term followup outcomes. Results: A total of 141 procedures were performed in 82 patients (17 underaged and 65 adult patients). The ERCP indications included abdominal pain (39.02\%), pancreatitis (12.20\%), recurrent pancreatitis (36.59\%), and other discomfort (12.20\%). The endoscopic interventions included endoscopic pancreatic sphincterotomy in $44.68 \%$ of the patients, bouginage in $26.95 \%$, pancreatic ductal stone extraction in 19.15\%, endoscopic nasopancreatic drainage in $21.99 \%$, and endoscopic retrograde pancreatic drainage in $56.74 \%$. After a median follow-up of 41 months, the overall response rate was $62.32 \%$. Between the underaged group and the adult group, significant differences were not observed in the ERCP procedures, complications and longterm follow-up results. Conclusions: ERCP is a safe and effective treatment for symptomatic PD. Based on the details, complications, and follow-up results, the ERCP procedure did not present differences between the underaged and adult groups. (Gut Liver 2016;10:476-482)

Key Words: Pancreas divisum; Endoscopic retrograde cholangiopancreatography; Endoscopic pancreatic sphincter- otomy; Endoscopic nasopancreatic drainage; Endoscopic retrograde pancreatic drainage

\section{INTRODUCTION}

Pancreas divisum (PD), whose prevalence is reported to be from $2.7 \%$ to $22 \%,{ }^{1,2}$ is the most common congenital anomaly of the pancreas. PD results from the nonfusion of the dorsal and ventral pancreatic ducts during embryologic development, and it can be divided into complete and incomplete PD. The former has a completely separate pancreatic duct system, while the latter has an insufficient communication between the ventral and dorsal pancreatic ducts. Usually it is asymptomatic, ${ }^{3}$ however, in a minority of patients, abdominal pain, acute pancreatitis (AP), and recurrent acute pancreatitis (RAP) would occur, which seriously affect a patient's quality of life. ${ }^{4,5}$

The treatment of PD remains controversial, but with the advancement in endoscopic retrograde cholangiopancreatography (ERCP) techniques, the procedures not only present as the gold standard for diagnosing PD, but they also exert their power in the therapy of PD, aiming to relieve pain, and to slow down the progress of chronic pancreatitis (CP). Nonetheless, the relevant articles in the literature are scarce, and the sample size of the trials is small and the details of ERCP have not been sufficiently reported. Herein, we carried out this long-term follow-up study to evaluate the efficacy and safety of ERCP in dealing with symptomatic PD. On the other hand, PD is a congenital disease; it can be detected at any age, from children to adults. As we all know, some of the body functions of the children may not be well-developed, and that they tended to be more likely to be injured during invasive procedures. Whether endoscopic in-

Correspondence to: Biao Gong

Digestive Endoscopy Center, Department of Gastroenterology, Ruijin Hospital, Shanghai Jiaotong University School of Medicine, 197 Ruijin Er Road, Shanghai 200025, China

Tel: +86-21-64370045600926, Fax: +86-21-64370045600926, E-mail: gbercp616@163.com

Received on August 1, 2015. Revised August 16, 2015. Accepted on August 16, 2015. Published online January 21,2016

Yi Lu and Bin Xu contributed equally to this work as first authors.

pISSN 1976-2283 eISSN 2005-1212 http://dx.doi.org/10.5009/gnl15362

(c) This is an Open Access article distributed under the terms of the Creative Commons Attribution Non-Commercial License (http://creativecommons.org/licenses/by-nc/4.0) which permits unrestricted non-commercial use, distribution, and reproduction in any medium, provided the original work is properly cited. 
tervention via ERCP would do more harm to children with PD, and whether the benefits the children obtain are the same as the adults have not been evaluated. So another aim of this study was to discuss whether ERCP procedures and results, in the underaged group were different from those of adults.

\section{MATERIALS AND METHODS}

\section{Patients}

From January 2008 to May 2014, consecutive patients with symptomatic PD and those who received endotherapy were included in the study. The patients selected had to meet the following criteria: (1) to be symptomatic and requiring intervention; (2) magnetic resonance cholangiopancreatography suggesting PD and proved by ERCP; (3) the patients received ERCP treatment; and (4) relevant medical data could be obtained. The exclusion criteria were (1) therapeutic ERCP was not performed, or not performed in our center; (2) poor physical status that could not tolerate ERCP procedure, or the risks outweighed the benefits; and (3) pertinent medical data was not available. The patients were divided into two groups based on their age: the underaged group (age $<18$ years) and the adult group (age $\geq 18$ years). A manual chart review was performed to acquire the fol- lowing data: age, gender, symptoms, past histories, laboratory test results, imaging results, ERCP operative details, and status after ERCP. The patients were then contacted by telephone and by searching the medical records in our hospital to get the longterm follow-up outcomes. Patients were asked if their symptoms were relieved after endotherapy; or if AP ever occurred; if they had to take other medicine or surgeries to relieve the symptoms; and if they were content with the endotherapy. The study protocol was approved by the Institutional Review Board in RuiJin Hospital.

\section{ERCP procedures}

Informed written consent for the ERCP procedure was obtained from the patient or from the parent or guardian of each child. The patients were sedated with intravenous diazepam and/or pethidine, or neither based upon the anaesthetist's judgment. The ERCP was performed with a side-viewing duodenoscope (TJF-240, TJF-260, JF-260; Olympus Corp., Tokyo, Japan) by experienced endoscopists. Additional cannulation of the minor papilla was performed if the entire length of the main duct was not detected by cannulation of the major papilla alone. According to the image of pancreatogram, the endoscopists would then perform endoscopic pancreatic sphincterotomy
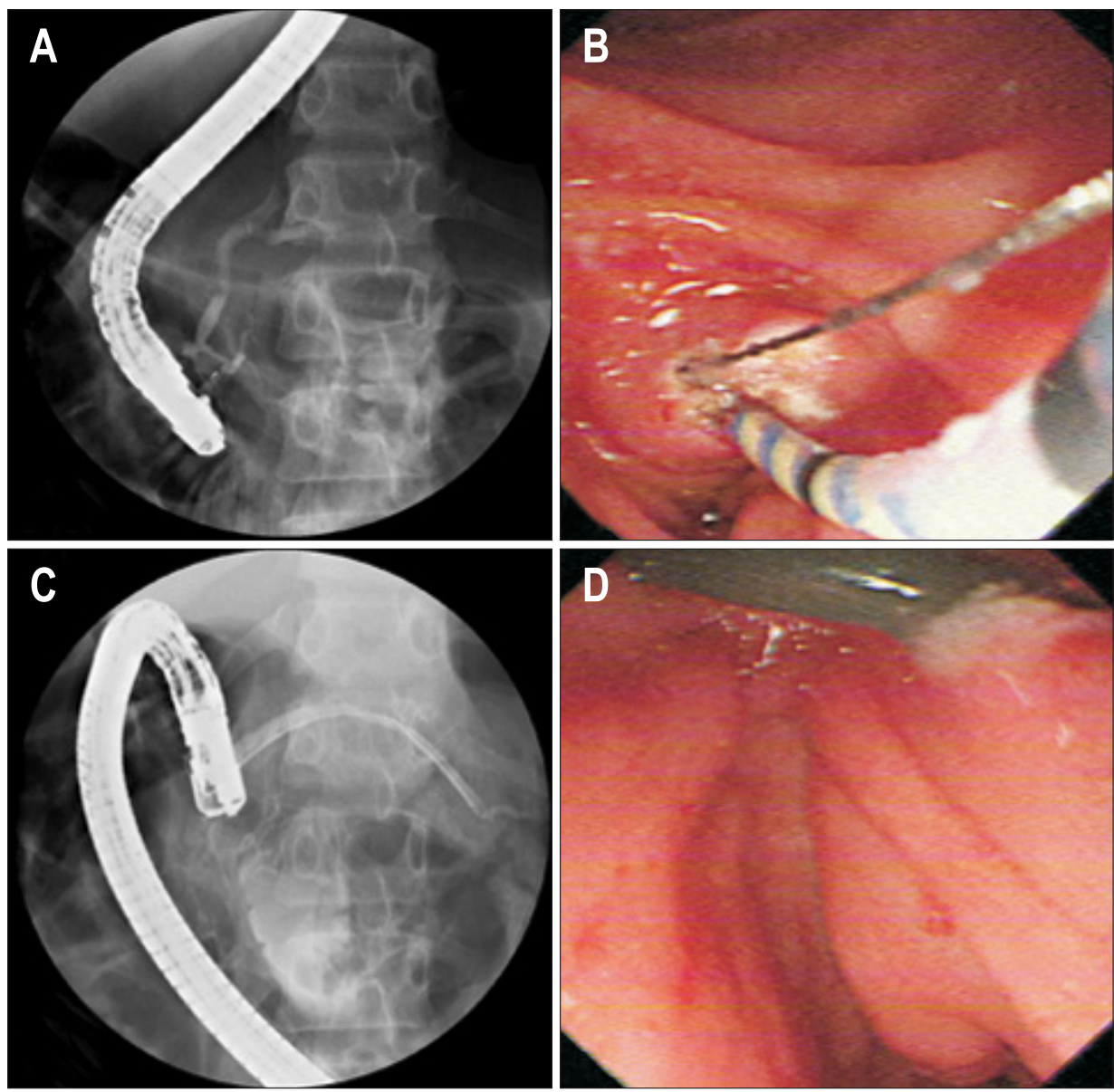

Fig. 1. (A) Fluoroscopic view of the endoscopic retrograde cholangiopancreatography showing a small ventral pancreatic duct and large dorsal pancreatic duct with dilation after contrast injection. (B) Endoscopic view of pancreatic sphincterotomy through the minor papilla. (C) Fluoroscopic view of the endoscopic retrograde pancreatic drainage. (D) Endoscopic view showing that a pancreatic stent was placed through the minor papilla. 
(EPS), bouginage, pancreatic ductal stone extraction, endoscopic nasopancreatic drainage (ENPD), or endoscopic retrograde pancreatic drainage (ERPD) to relieve the patient's symptoms. Fig. 1 shows the process of EPS and ERPD in the same patient. The endoscopists would use a 4-point scale to evaluate how difficult the procedure was: 1 , without difficulty; 2 , a little difficult; 3 , difficult; 4, very difficult.

\section{Outcome parameters}

The primary outcome parameters were the success rate and response rate. Success rate was defined as the rate of technical success of ERCP, which meant that the endoscopists had successfully performed the ERCP procedures that they had planned. Response rate was the long-term follow-up result. At the time of last follow-up, we used a 5-point Likert scale to evaluate the patients' abdominal pain: 1, cured; 2, better; 3, same; 4, worse; and 5, much worse, which subsequently meant that surgery was required. If the point score was 1 or 2, then it was defined as relief. Response was defined as pain relief and without recurrence of pancreatitis.

The secondary outcome parameters were the indications for ERCP, the ERCP procedure details, and the post-ERCP complications. Complications were assessed on the basis of the consensus criteria. ${ }^{6,7}$ Post-ERCP pancreatitis (PEP) was defined as abdominal pain persisting for more than 24 hours, with serum amylase levels elevated to more than 3 times of the upper limit of normal. Infection was defined as a fever that was higher than $38^{\circ} \mathrm{C}$ and that lasted for longer than 24 hours. Hemorrhage could happen during and after the process of ERCP.

\section{Statistical analysis}

SAS version 8.2.0 (SAS Institute Inc., Cary, NC, USA) was used to perform the statistical analyses; continuous variables with a normal distribution were tested by the Student t-test; otherwise the nonparametric Wilcoxon rank-sum test was used. The categorical variables were tested by using chi-square test with Yates correction or with Fisher exact test. Statistical significance was defined as $\mathrm{p}<0.05$ (two-tailed).

\section{RESULTS}

\section{Patients}

A total of 141 procedures were performed in 82 patients, of whom 17 patients (37 procedures) were underaged, and 65 patients (104 procedures) were adults. The demographic details of the two groups are presented in Table 1. The ERCP indications for PD included abdominal pain (32/82, 39.02\%), AP (10/82, 12.20\%), RAP (30/82, 36.59\%) and other discomforts (10/82, $12.20 \%$ ), with no difference in both groups. It seemed that patients with PD were more likely to be complicated with other pancreaticobiliary malformations or tumors. Five patients had pancreaticobiliary maljunction (among whom, one also had pancreatic mucinous cystic neoplasm, and another one had gallbladder carcinoma), three patients had low confluence of cystic duct, one patient had low confluence of right and left hepatic ducts, one patient had duodenal diaphragm, two patients had duodenal adenoma, four patients suffered with intraductal papillary mucinous neoplasm (IPMN), and two patients had pancreatic cancer.

\section{ERCP procedures}

Altogether, 141 procedures were performed, and 30 patients (36.59\%) required more than one procedure. The total success rate was $91.1 \%$ and the success rates in both groups were similar. The reason for unsuccessful outcome was mainly failure after repetitive cannulation to the pancreatic duct. Based on the images of the pancreatogram, together with clinical manifestations, 62 patients (75.61\%) were diagnosed as suffering from $\mathrm{CP}$ at the same time. Pancreatic duct dilation was found in 109 procedures (77.30\%), and pancreatic duct stricture was found in 38 procedures (26.95\%). In general, most of the procedures were evaluated as not being difficult, and 37 procedures (26.24\%)

Table 1. Baseline Characteristics of Patients with Pancreas Divisum Who Underwent Endoscopic Retrograde Cholangiopancreatography

\begin{tabular}{lccc}
\hline \multicolumn{1}{c}{ Characteristic } & Underaged $(\mathrm{n}=17)$ & Adult $(\mathrm{n}=65)$ & $\mathrm{p}$-value \\
\hline Age, yr & $9(2-16)$ & $51(19-87)$ & $<0.0001$ \\
Sex, male:female & $6: 11$ & $36: 29$ & 0.1401 \\
Indications & & & 0.7232 \\
$\quad$ Abdominal pain & $6(35.5)$ & $26(40.0)$ & 1.0000 \\
$\quad$ Acute pancreatitis & $2(11.8)$ & $8(12.3)$ & 0.3139 \\
$\quad$ Recurrent acute pancreatitis & $8(47.1)$ & $22(33.9)$ & 0.6791 \\
$\quad$ Other discomfort & $1(5.9)$ & $9(13.9)$ & 1.0000 \\
Other pancreaticobiliary malformation/disease & $4(23.5)$ & $14(21.5)$ & 0.1119 \\
Diabetes mellitus & 0 & $10(15.4)$ & 0.9024 \\
Follow-up duration, mo & $43.5(10-76)$ & $41(5-76)$ & \\
\hline
\end{tabular}

Data are presented as the median (range) or number (\%). 
were scored as difficult or very difficult. In total, endoscopic interventions included EPS in 63 procedures (44.68\%), bouginage in 38 (26.95\%), pancreatic ductal stones extraction in 27 (19.15\%), ENPD in 31 (21.99\%), and ERPD in 81 (57.45\%). The stents used ranged from 5 to $8.5 \mathrm{Fr}$ in diameter and 3 to 12 $\mathrm{cm}$ in length. In 46 procedures (32.62\%), EPS was performed through the minor papilla and in 28 procedures (19.86\%) through the major papilla. ERPD was performed through the minor papilla in 81 procedures (57.45\%) and in 44 procedures (31.21\%) through the major papilla. The above procedures made no statistical difference in both groups. Fig. 2 summarizes the details of the procedures.

\section{Complications}

Fourteen patients (9.93\%) had pancreatitis (10, mild; 3, moderate; 1 , severe), and the patients (except for the severe one) recovered through conservative treatment, requiring prolongation of hospital stay by 2 to 6 days. The patient (26 years old) who developed severe PEP was on his course of AP at admission (also complicated with systemic lupus erythematosus); he was then transferred to the intensive care unit, and recovered after surgery. Five patients (3.55\%) had biliary infection, each lasting for no more than 3 days. One child (0.71\%) had moderate hemorrhage after EPS, and emergent gastroduodenoscopy revealed that the hemorrhage came from the incision of the minor papilla. After fluid infusion, blood transfusion, and haemostasis therapy, the child eventually recovered. The respective complication rates for the underaged and adult group are shown in Table 2. No statistical difference was found between the two groups.

\section{Follow-up results}

During a median follow-up of 41 months (range, 5 to 76 months), 13 patients (15.85\%) were lost to follow-up; two were

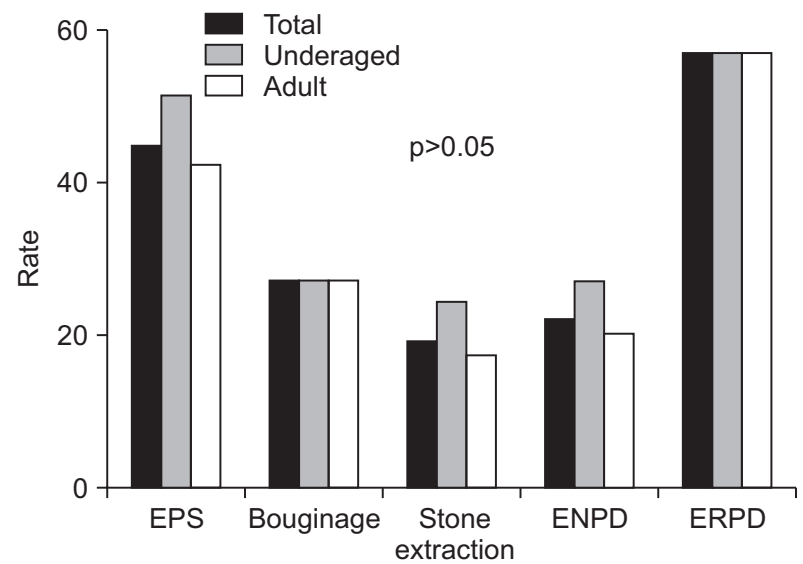

Fig. 2. Details of the endoscopic retrograde cholangiopancreatography procedures for pancreas divisum.

EPS, endoscopic pancreatic sphincterotomy; ENPD, endoscopic nasopancreatic drainage; ERPD, endoscopic retrograde pancreatic drainage. in the underaged group and 11 were in the adult group. After one or more ERCP procedures, 54 patients (78.26\%) were reported to have experienced relief, which meant that they reflected that the frequency and the degree of their abdomina pain was better than before, or had even disappeared. Twelve patients (17.39\%) still complained of pancreatitis after ERCP (but they indicated that the severity of the pancreatitis was milder than before). The overall response rate was 62.32\% (43 in 69 patients). Six patients (8.70\%) needed to take pancreatic enzyme drugs as a supplement for digestion. Follow-up results of the two groups had no statistical difference (Table 2). Altogether five patients received surgery, and the reason, type of the surgery and the follow-up results are shown in Table 3.

\section{DISCUSSION}

The pathogenesis of PD is that the ventral and dorsal pancreatic ducts failed to fuse during the eighth week of the fetus development. As a result, the Santorini's duct becomes the major drainage channel, which drains the pancreatic fluid through the

Table 2. Details of the Endoscopic Retrograde Cholangiopancreatography Procedures and Long-Term Follow-Up Results of the Patients with Pancreas Divisum

\begin{tabular}{|c|c|c|c|}
\hline Detail & $\begin{array}{l}\text { Underaged } \\
\qquad(\mathrm{n}=17)\end{array}$ & $\begin{array}{l}\text { Adult } \\
(n=65)\end{array}$ & $\mathrm{p}$-value \\
\hline No. of procedures & 37 & 104 & - \\
\hline Success procedures & $32(86.5)$ & $96(92.3)$ & 0.3260 \\
\hline Degree of difficulty & $1(1-4)$ & $1(1-4)$ & 0.1377 \\
\hline Chronic pancreatitis & $15(88.2)$ & 47 (72.3) & 0.2188 \\
\hline Complete pancreas divisum & $10(58.8)$ & 47 (72.3) & 0.2823 \\
\hline Pancreatic duct dilation & $15(88.2)$ & $45(69.2)$ & 0.1373 \\
\hline Pancreatic duct stricture & $13(35.1)$ & $25(24.0)$ & 0.1914 \\
\hline EPS through minor papilla & $11(29.7)$ & 35 (33.7) & 0.6619 \\
\hline EPS through major papilla & $9(24.3)$ & 19 (18.3) & 0.4278 \\
\hline ERPD through minor papilla & $20(54.1)$ & $61(58.7)$ & 0.6270 \\
\hline ERPD through major papilla & $12(32.4)$ & $32(30.8)$ & 0.8512 \\
\hline \multicolumn{4}{|l|}{ Complications } \\
\hline Pancreatitis & $3(8.1)$ & $11(10.6)$ & 1.0000 \\
\hline Infection & 0 & $5(4.8)$ & 0.3258 \\
\hline Hemorrhage & $1(2.7)$ & 0 & 0.2624 \\
\hline Hospital stay, day & $9(4-20)$ & $9(3-163)$ & 0.6168 \\
\hline \multicolumn{4}{|l|}{ Follow-up results } \\
\hline Follow-up loss & $2(11.8)$ & $11(16.9)$ & 1.0000 \\
\hline Pain relief & 15 (100.0) & 39 (72.22) & 0.3155 \\
\hline Pancreatitis & $5(33.3)$ & $7(13.0)$ & 0.2630 \\
\hline Response rate & $10(66.7)$ & $33(61.1)$ & 0.5223 \\
\hline
\end{tabular}

Data are presented as the median (range) or number (\%). EPS, endoscopic pancreatic sphincterotomy; ERPD, endoscopic retrograde pancreatic drainage. 
Table 3. Details of the Reason, Surgery Type and Follow-Up Results

\begin{tabular}{cclll}
\hline Patient & Age, yr & \multicolumn{1}{c}{ Reason } & \multicolumn{1}{c}{ Type } & Follow-up results \\
\hline HZL & 56 & Pancreatic carcinoma & Abdominal laparotomy & Death \\
LMJ & 56 & Gallbladder carcinoma & Cholecystectomy+biopsy of liver & Death \\
ZRT & 54 & Intolerability of pain & Pancreaticoduodenectomy & Persistent abdominal pain \\
WSG & 41 & Intolerability of pain & Pancreaticoduodenectomy & Pain relieved \\
WRX & 52 & Pancreatic pseudocyst & Pain relieved & \\
\hline
\end{tabular}

minor papilla, in contrast to the Wirsung's duct, which assumes a minor role. ${ }^{8-10}$ This change is totally opposite to the normal condition, as the orifice of the minor papilla is much smaller, while it assumes the major task; consequently, pancreatic hypertension would occur. Most patients may be asymptomatic or just exhibit proof of dilation of the pancreatic ducts, some may present pancreatic abdominal pain, or fluctuation of serum amylase, or onset of AP; and in some cases, CP would occur. The clinical difference of PD has been a controversy for many years. In the present study, we chose the patients with symptomatic PD, who had also received endoscopic therapy, and made an analysis on their clinical characteristics, specific ERCP procedures, and long-term follow-up results.

We found that $39.02 \%$ of the selected patients had abdominal pain, 12.20\% had AP, 36.59\% had RAP, and 75.61\% were found to suffer from CP. Compared with other trials, ${ }^{11,12}$ the indications for patients with PD to receive ERCP are similar, with small difference in proportion.

When reviewing the medical records of these patients, we conceived that there was an inclination that those patients with PD had a greater risk of other pancreaticobiliary abnormalities, such as pancreaticobiliary maljunction, IPMN, pancreaticobiliary cancer, and so forth. With great interests, we searched through the related articles in the literature and discovered that some case reports told us the same story. ${ }^{13-15}$ One retrospective study conducted by Takuma et al., ${ }^{16}$ showed that of the 54 patients with complete PD, six patients (11.10\%) were found to have pancreatic tumours and that all tumours developed from the dorsal pancreas. They deduced that in complete PD the dorsal pancreatic duct might be a factor that promotes oncogenesis. Whether this is a coincidence or not still needs more studies to clarify the situation. We also need to figure out which genes might play roles in the pancreas anomaly, and whether these genes are associated with oncogenesis or how malformation correlates with oncogenesis and whether other factors might have an impact on this process.

Endoscopic treatments for symptomatic PD include EPS, ERPD, and ENPD, in order to improve the drainage of pancreatic ducts, so as to decompress the pancreatic pressure. If $\mathrm{CP}$ develops and pancreatic stones are formed, then extracorporeal shock wave lithotripsy and pancreatic ductal stone extraction would be needed. ${ }^{17}$ Articles describing specific procedure-related details during therapeutic ERCP are limited. Rustagi and Golioto ${ }^{11}$ depicted 45 patients' ERCP details, and in their study, $42.4 \%$ of the patients received dorsal sphincterotomy, 82.2\% received ERPD, and 18.9\% required stricture dilation or balloon sweeping. In Yamamoto et al.'s study, ${ }^{18} 50 \%$ of the patients received ERPD. The ERCP procedure details in the present study were not widely divergent to their result.

Just like in other diseases, ERCP procedure would bring about adverse events, and in this study, the incidences for PEP, infection, and hemorrhage were 9.93\%, 3.55\%, and 0.71\%, respectively. Fortunately, most of them were in mild to moderate degrees. Compared with the former study conducted by ourselves ${ }^{19}$ (which compared the safety and the efficacy of sphincterotomy and balloon dilation in common bile duct stone removal), it was apparent that the incidence of PEP for PD had increased, as it was $2.52 \%$ in the sphincterotomy group and $7.05 \%$ in the balloon dilation group, while for the rates of infection and hemorrhage, the difference was not obvious. This data was similar to the study of Chacko et al. ${ }^{12}$ which reported $10.68 \%$ for PEP and $0.97 \%$ for perforation. Other trials which indicate the information of adverse events after ERCP for patients with PD are still lacking. The risks for patients with PD to develop PEP seem to be greater, and it is not difficult to understand, since that pancreatic duct injection, pancreatic sphincterotomy, and nonprophylactic pancreatic duct stents, are all risk factors for PEP. ${ }^{20,21}$

The long-term outcome of endotherapy has been the focus of many researchers. A number of trials have assessed the efficacy of endotherapy in PD, and most of them have divided the patients into different groups, according to their indications: RAP, $\mathrm{CP}$, and simple abdominal pain. A systematic review conducted in 2008 summarized all of the associated articles published from 1950 to May 2008, ${ }^{22}$ and exhibited that the pooled overall response rates to endotherapy were $69.4 \%$, and the respective rates for RAP, $\mathrm{CP}$, and pain type $\mathrm{PD}$ were $81.2 \%, 68.8 \%$, and $53.1 \%$, respectively. The newest systematic review in 2014 gave a response rate of 76\%, 42\%, and 33\% for RAP, CP, and pain, respectively. ${ }^{23}$ From the above studies, we may draw the conclusion that, the response rates of endotherapy for PD are different regarding different indications, and thus, the overall rate is influenced by the proportion of each categories of patients.

For symptomatic PD, whether surgery is a superior procedure than endotherapy remains unknown, as it appears to be unreal- 
istic to carry out prospective, randomized, and controlled trials. In 2008 , Liao et al. ${ }^{22}$ conducted a systematic review to compare the efficacy of endotherapy and surgery for PD, and they concluded that the pooled response rates of patients with PD had no statistical difference towards the choice of endotherapy or surgery, and they deduced that at present, endotherapy was still a reasonable first-line option for PD, depending on the patient's preference and its less invasiveness.

Being a congenital disease, PD can be found at almost any age. The youngest patient in the present study was 2 years old, and 17 patients were underaged. ERCP procedure appears to be technically difficult in the underaged, ${ }^{24}$ thus we made a further analysis on whether the ERCP in the underaged was different from that of the adult. The results showed that the indications, success rate, specific procedures, adverse events, and followup results had no difference in the two groups. Rustagi and Golioto $^{11}$ also did not find any difference associated with age, and Agarwal et al. ${ }^{24}$ concluded that, in managing pancreatic diseases, the ERCP-related adverse event rates in children were comparable to those in adults. While Borak et al.'s multivariate analysis $^{25}$ showed that in RAP and CP type patients, older ages were found to have higher response rates. We did not observe similar results as Borak et al. ${ }^{25}$ did, possibly due to the relatively small sample size of the underaged group.

Although PD is the most common variant of the pancreas, and the number of related publications has reached to be more than 700 , nearly $25 \%$ of these were case reports. ${ }^{22}$ Most of the remaining articles are retrospective studies, and only a small sample-sized randomized controlled trial involving 19 patients with RAP, was published. ${ }^{26}$ Among the existing articles, the specific ERCP details and the risks of ERCP-related adverse events, are not well established or reported, and the long-term outcome of endotherapy for PD is perhaps the least studied, ${ }^{27}$ thus we conducted this survey so as to add some data to the limited literature. The main limitations of this study are its retrospective and single-centered nature, and hence, these results may not be generalizable to all of the units. Secondly, as we visited the patients by telephone and observed the medical records, the recall bias and the loss to follow-up bias cannot be excluded. Thirdly, we did not group and analyze the patients according to their indications as other trials did. The main reason was that if we had used the definitions of the three categories as the previous trials did, then there would have been only six patients in the RAP group, while there would have been 62 patients in the $\mathrm{CP}$ group, and 14 patients in the pain group. We thought that it was meaningless to do so at this time, but if we accumulate more patients, maybe in the future, we can analyze those patients based on their indications. Prospective trials with greater sample sizes and longer follow-up periods are still needed.

In conclusion, in symptomatic patients with PD, ERCP is safe, although with a higher rate for PEP (in this study, most were in a mild to moderate degree). Patients can achieve good outcomes after endotherapy, however, some will require multiple procedures. The ERCP details, adverse events, and follow-up results, had no difference between the underaged group and the adult group. The relationship between PD and other pancreaticobiliary malformations and tumours still needs further exploration.

\section{CONFLICTS OF INTEREST}

No potential conflict of interest relevant to this article was reported.

\section{ACKNOWLEDGEMENTS}

The authors thank all the staffs from the Digestive Endoscopy Center, Ruijin Hospital, Shanghai Jiaotong University School of Medicine for their help in this study.

\section{REFERENCES}

1. Kin T, Shapiro AM, Lakey JR. Pancreas divisum: a study of the cadaveric donor pancreas for islet isolation. Pancreas 2005;30:325327.

2. Alempijevic T, Stimec B, Kovacevic N. Anatomical features of the minor duodenal papilla in pancreas divisum. Surg Radiol Anat 2006;28:620-624.

3. Brugge WR. Endoscopic approach to the diagnosis and treatment of pancreatic disease. Curr Opin Gastroenterol 2013;29:559-565.

4. Klein SD, Affronti JP. Pancreas divisum, an evidence-based review: part I, pathophysiology. Gastrointest Endosc 2004;60:419 425.

5. Khalid A, Slivka A. Approach to idiopathic recurrent pancreatitis. Gastrointest Endosc Clin N Am 2003;13:695-716.

6. Cotton PB, Lehman G, Vennes J, et al. Endoscopic sphincterotomy complications and their management: an attempt at consensus. Gastrointest Endosc 1991;37:383-393.

7. Cotton PB. Outcomes of endoscopy procedures: struggling towards definitions. Gastrointest Endosc 1994;40:514-518.

8. Kamisawa T. Clinical significance of the minor duodenal papilla and accessory pancreatic duct. J Gastroenterol 2004;39:605-615.

9. Cohen SA, Siegel JH. Pancreas divisum: endoscopic therapy. Surg Clin North Am 2001;81:467-477.

10. Kozu T, Suda K, Toki F. Pancreatic development and anatomical variation. Gastrointest Endosc Clin N Am 1995;5:1-30.

11. Rustagi T, Golioto M. Diagnosis and therapy of pancreas divisum by ERCP: a single center experience. J Dig Dis 2013;14:93-99.

12. Chacko LN, Chen YK, Shah RJ. Clinical outcomes and nonendoscopic interventions after minor papilla endotherapy in patients with symptomatic pancreas divisum. Gastrointest Endosc 2008;68: 667-673.

13. Santi L, Renzulli M, Patti C, Cappelli A, Morieri ML. First case of 2 intraductal papillary mucinous tumors of both ventral and dorsal ducts in pancreas divisum. Pancreas 2010;39:110-111. 
14. Kamisawa T, Tu Y, Egawa N, Tsuruta K, Okamoto A, Matsukawa M. Pancreas divisum in pancreaticobiliary maljunction. Hepatogastroenterology 2008;55:249-253.

15. Nakamura Y, Tajiri T, Uchida E, et al. Adenoma of the minor papilla associated with pancreas divisum. Hepatogastroenterology 2007;54:1841-1843.

16. Takuma K, Kamisawa T, Tabata T, Egawa N, Igarashi Y. Pancreatic diseases associated with pancreas divisum. Dig Surg 2010;27:144148.

17. Tandan M, Nageshwar Reddy D. Endotherapy in chronic pancreatitis. World J Gastroenterol 2013;19:6156-6164.

18. Yamamoto N, Isayama H, Sasahira N, et al. Endoscopic minor papilla balloon dilation for the treatment of symptomatic pancreas divisum. Pancreas 2014;43:927-930.

19. Lu Y, Wu JC, Liu L, Bie LK, Gong B. Short-term and long-term outcomes after endoscopic sphincterotomy versus endoscopic papillary balloon dilation for bile duct stones. Eur J Gastroenterol Hepatol 2014;26:1367-1373.

20. Wong LL, Tsai HH. Prevention of post-ERCP pancreatitis. World J Gastrointest Pathophysiol 2014;5:1-10.

21. Chen JJ, Wang XM, Liu XQ, et al. Risk factors for post-ERCP pancreatitis: a systematic review of clinical trials with a large sample size in the past 10 years. Eur J Med Res 2014;19:26.

22. Liao Z, Gao R, Wang W, et al. A systematic review on endoscopic detection rate, endotherapy, and surgery for pancreas divisum. Endoscopy 2009;41:439-444.

23. Kanth R, Samji NS, Inaganti A, et al. Endotherapy in symptomatic pancreas divisum: a systematic review. Pancreatology 2014;14: 244-250.

24. Agarwal J, Nageshwar Reddy D, Talukdar R, et al. ERCP in the management of pancreatic diseases in children. Gastrointest Endosc 2014;79:271-278.

25. Borak GD, Romagnuolo J, Alsolaiman M, Holt EW, Cotton PB. Long-term clinical outcomes after endoscopic minor papilla therapy in symptomatic patients with pancreas divisum. Pancreas 2009;38:903-906.

26. Lans JI, Geenen JE, Johanson JF, Hogan WJ. Endoscopic therapy in patients with pancreas divisum and acute pancreatitis: a prospective, randomized, controlled clinical trial. Gastrointest Endosc 1992;38:430-434.

27. Ahmed F, Sherman S. Minor papilla endotherapy in patients with symptomatic pancreas divisum. Gastrointest Endosc 2008;68:674675. 